\title{
Artificial Liver Support System Improves Short-Term Outcomes of Patients with HBV-Associated Acute-on-Chronic Liver Failure: A Propensity Score Analysis
}

\author{
Lan-Lan Xiao, Xiao-Wei Xu, Kai-Zhou Huang, Ya-Lei Zhao, Ling-Jian Zhang, \\ and Lan-Juan Li \\ State Key Laboratory for Diagnosis and Treatment of Infectious Diseases, \\ Nationwide Clinical Research Center for Infectious Diseases, \\ Collaborative Innovation Center for Diagnosis and Treatment of Infectious Diseases, The First Affiliated Hospital, \\ College of Medicine, Zhejiang University, Hangzhou, China \\ Correspondence should be addressed to Lan-Juan Li; 1jli@zju.edu.cn
}

Received 18 September 2019; Accepted 9 November 2019; Published 29 November 2019

Academic Editor: Haruki Komatsu

Copyright (c) 2019 Lan-Lan Xiao et al. This is an open access article distributed under the Creative Commons Attribution License, which permits unrestricted use, distribution, and reproduction in any medium, provided the original work is properly cited.

Background. Hepatitis B virus-associated acute-on-chronic liver failure (HBV-ALCF) is a complicated syndrome with extremely high short-term mortality. The artificial liver support system (ALSS) may improve the liver function for patients with HBV-ACLF, but the data on its short-term outcomes are insufficient in China. Methods. We recruited HBV-ACLF patients in this nationwide, multicenter, retrospective study. Patients with HBV-ACLF were diagnosed by the COSSH-ACLF criteria. Propensity score matching (PSM) analysis was used to generate compared pairs. The short-term (28/90 days) survival rates between the standard medical therapy (SMT) group and ALSS group were calculated using a Kaplan-Meier graph. Result. In total, 790 patients with HBV-ACLF were included in this retrospective study; 412 patients received SMT only (SMT group), and 378 patients received SMT and ALSS treatment (ALSS group). PSM generated 310 pairs and eliminated the baseline differences between the two groups ( $p>0.05$ for all baseline variables). The probabilities of survival on day 28 were $65.2 \%(205 / 310)$ in the ALSS group and 59.0\% $(185 / 310)$ in the SMT group; on day 90 , they were $51.0 \%$ (163/310) and $42.3 \%$ (136/310). The short-term (28/90 days) survival rates of the ALSS group were significantly higher than those of the SMT group ( $p=0.0452$ and $p=0.0187$, respectively). Compared to receiving SMT alone, treatment with ALSS was associated with a significant reduction in serum bilirubin levels and the model for end-stage liver disease (MELD) scores at day 7 and day 28. Multivariate logistic regression analysis revealed that older age, high total bilirubin (T-Bil), low albumin, high ALT, high MELD scores, and high COSSH-ACLF grade were independent baseline factors associated with poor prognosis. Conclusions. This retrospective study found that compared to SMT, the ALSS improved the short-term (28/90 days) survival rates and laboratory parameters in HBV-ACLF patients. The ALSS had a better therapeutic effect than SMT for patients with HBV-ACLF in China.

\section{Introduction}

Chronic hepatitis $\mathrm{B}(\mathrm{CHB})$ is a major public health challenge in China, with an estimated 78 million chronic carriers and 28 million patients with active hepatitis [1]. $\mathrm{CHB}$ is a significant risk factor that accounts for nearly $45 \%$ of cases of hepatocellular carcinoma (HCC) and 30\% of cases of cirrhosis, causing nearly 1 million deaths each year worldwide $[2,3]$. The high prevalence of $\mathrm{CHB}$ causes that hepatitis B virus (HBV) infection absolutely predominated in the etiologies of ACLF, accounting for 96.5\% cases in China, while alcoholism is the most common etiologies of ACLF in western developed countries $[4,5]$.

HBV-related ACLF (HBV-ALCF) is a complicated syndrome with high short-term mortality (40-70\% without liver transplantation) that develops in patients with HBVrelated chronic liver disease $[6,7]$. 
In past decades, a series of artificial liver support systems (ALSSs) have been applied in liver failure which aim to detoxify blood and compensate liver function for patients with liver failure. Molecular adsorbent recirculating system (MARS) $[8,9]$ and Prometheus $[10,11]$ are the most widely used ALSS. Li's artificial liver system (Li-ALS, the low-volume plasma exchange-centered ALSS) was designed by Professor Li's team since 1986 [12], which is mainly used in China. Some studies demonstrated that ALSSs could detoxify and ameliorate hepatic encephalopathy during acute liver failure (ALF) [11, 13, 14]. However, several large randomized trials noted that patients with ALF [15] or ACLF [16, 17], supported with ALSSs, did not show an increased short-term survival. A single-center study conducted in China reported that ALSS improved 90 days and 5 years outcomes of patients with HBV-ACLF [18].

In this study, we conducted a nationwide, multicenter, retrospective study to test whether ALSSs could improve the short-term (28/90 days) outcomes in patients with HBVALCF in China and identify predictive factors for the prognosis of these patients.

\section{Methods}

2.1. Patients. This is a retrospective cohort study where all data were fully anonymized before access. We recruited hospitalized patients with HBV-ACLF from 11 liver centers of Chinese University hospitals between January 2014 and May 2017. The following clinical data were collected: demographic data (age, sex, and body mass index), cirrhosis, laboratory measurements (HBV-DNA level, ALT, total bilirubin (T-Bil), INR, and creatinine), hepatic encephalopathy (HE), nucleos(t)ide analogs (NA), and survival time. The liver disease severity was assessed using the MELD scores and COSSH-ACLF grade (Chinese Group on the Study of Severe Hepatitis B-ACLF). The study was approved by the Ethics Committee of the First Affiliated Hospital, Zhejiang University School of Medicine.

2.2. Inclusion and Exclusion Criteria. The inclusion criteria were as follows:

Patients diagnosed with $\mathrm{CHB}$ [19] (HBV surface antigenpositive $\geq 6$ months; serum HBV-DNA $\geq 20000 \mathrm{IU} / \mathrm{mL}$; a liver biopsy showing chronic hepatitis) and reached at least ACLF grade 1 diagnosed by the COSSH-ACLF criteria [6]:

(1) ACLF grade 1: patients with kidney failure alone; patients with single liver failure (total bilirubin $\geq 12 \mathrm{mg} / \mathrm{dL}$ ) with an international normalized ratio (INR) $\geq 1.5$ and/or kidney dysfunction and/or $\mathrm{HE}$ grade I or II; patients with single type of organ failure of the coagulation, circulatory, or respiratory systems and/or kidney dysfunction and/or HE grade I or II; and patients with cerebral failure alone plus kidney dysfunction

(2) ACLF grade 2: patients with failure of two organ systems
(3) ACLF grade 3: patients with failure of 3 or more organ systems

The exclusion criteria were as follows:

(1) Younger than 18 years or older than 80 years

(2) Received a liver transplant

(3) Human immunodeficiency virus infection

(4) Diagnosed with HCC or another tumor

(5) Dead within 3 days

(6) Had a severe comorbidity that could affect survival

2.3. Therapies. According to the Diagnostic and Treatment Guideline for Liver Failure 2012 (Guideline (2012)) [20], the treatment for severe liver disease mainly consists of restoring and preserving vital organ function and slowing down the progression of multiple organ failure. The standard medical therapy (SMT) included a high-calorie diet; enteral nutrition is recommended; correction hypoproteinemia; correction water-electrolyte and acid-base balance; nucleoside analogs for $\mathrm{HBV}$-DNA-positive patients; anti-infective therapy for infection; restricted protein diet; lactulose, ammonia drugs, and L-ornithine aspartate for HE; diuretics and tolvaptan for ascites; tubular active drugs; maintenance of arterial blood pressure and water restriction for hepatorenal syndrome; and oxygen therapy for hepatopulmonary syndrome.

According to the Guideline (2012), patients with earlyor middle-stage liver failure were advised to receive ALSS treatment. For patients with early liver failure, PE was applied; for patients with metaphase hepatic failure, continuous blood purification (CBP) was applied; for patients with brain edema or renal failure or imbalance of water and electrolytes, CBP or plasma diafiltration (PDF) was applied; for patients with hyperbilirubinemia, plasma bilirubin absorption (PBA) was applied. The ALSS sessions were scheduled as follows: the ALSS was usually performed in 48 hours after diagnosis. ALSS treatment was performed daily on the first 2 or 3 days; future treatments were offered according to the patients' condition. Overall, 841 ALSS treatment sessions were applied in 310 patients (average of 2.7 sessions per patient, ranging from 1 to 8 sessions). 9 (2.9\%) patients received $\mathrm{PE}(2-3 \mathrm{~h} /$ per session), and 301 (97.1\%) patients received continuous renal replacement therapy (CRRT, $8 \mathrm{~h}$ /per session).

2.4. Statistical Analysis. Propensity score matching analysis was used to eliminate bias between the two groups. Propensity scores were computed using the following variables: age; sex; serum levels of HBV-DNA, ALT, T-Bil, and albumin; platelet count; white cell count; creatinine; INR; serum sodium; cirrhosis; HE; MELD score; and COSSHACLF grade. For propensity score matching, a nearestneighbor 1:1 matching scheme was used. Categorical data were compared using the $\chi^{2}$ test or Fisher's exact test. Continuous variables were compared using the MannWhitney $U$ test. The survival rate at 28 days and 90 days were 
calculated using a Kaplan-Meier graph. The difference in the survival rate was compared using a log-rank test. The relationship between baseline parameters and 28-day survival was studied using a multivariate logistic regression model. All statistical analyses were performed using IBM SPSS v. 24.0 for Windows (IBM Corp., Armonk, NY, USA). $p$ values are two-tailed, and values less than 0.05 were considered statistically significant.

\section{Results}

3.1. Patient Characteristics. A total of 790 patients with HBV-ACLF were included in the study; 412 patients received standard treatment (SMT group) and 378 patients received ALSS treatment (ALSS group, Figure 1). Among them, 598 (85.3\%) patients were male and 369 (52.5\%) patients had no cirrhosis. Before matching, the baseline characteristics between two groups that differed significantly, such as ALT, T-Bil, and platelet count. Propensity score matching analysis generated 173 pairs, and the baseline characteristics of the pairs were balanced, with $p>0.05$ for all baseline variables. After matching, the mean (SD) age of the patients in the SMT and ALSS groups was 45.4 (11.1) and 46.8 (11.3) for male, and the mean (SD) MELD scores were 25.1 (5.2) and 24.9 (5.6). In the SMT group, 118 (38.1\%) patients had cirrhosis, and in the ALSS group, 133 (42.9\%) patients had cirrhosis. All patients received NAs after diagnosis. In the SMT group, 155 patients received $100 \mathrm{mg}$ of lamivudine (LAM) daily and 155 patients received $0.5 \mathrm{mg}$ of entecavir (ETV) daily. In the ALSS group, 131 patients received $100 \mathrm{mg}$ of LAM daily; 157 patients received $0.5 \mathrm{mg}$ of ETV daily; 14 patients received $300 \mathrm{mg}$ of tenofovir daily; and 8 patients received $600 \mathrm{mg}$ of telbivudine daily. The characteristics of all patients are shown in Table 1.

3.2. Survival Rates. For all patients, the short-term $(28 / 90$ days) survival rates were $60.0 \%(474 / 790)$ and $45.8 \%$ (362/ $790)$. The 28 -day survival rates were $65.2 \%(205 / 310)$ in the ALSS group and 59.0\% (185/310) in the SMT group; the 90day survival rates were $51.0 \%(163 / 310)$ and $42.3 \%(136 / 310)$, respectively. The short-term (28/90 days) mortality rate was significantly lower in the ALSS group than in the SMT group $(p=0.0452 ; p=0.0187$, Figures $2(a)$ and $2(b))$.

In the SMT group, patients treated with entecavir had similar short-term survival rates than patients treated with lamivudine $(p>0.05)$. In the ALSS group, patients treated with entecavir had higher short-term (28/90 days) survival rates than patients treated with lamivudine (77.1\%: $64.1 \%$; 62.4\%: 49.6\%, $p<0.05$, Table 2). The short-term (28/90 days) survival rates in the patients with noncirrhotic HBV-ACLF (63.6\%/49.65\%) and cirrhotic HBV-ACLF (61.8\%/46.2\%) were not significantly different.

The 28-day survival rates of patients with ACLF grades $1-3$ were $72.5 \%, 37.2 \%$, and 0 in the SMT group and $78.5 \%$, $43.5 \%$, and $11.1 \%$ in the ALSS group. The 90-day survival rates of patients with ACLF grades $1-3$ were $55.6 \%, 22.3 \%$, and 0 in the SMT group and $65.1 \%, 29.3 \%$, and $0 \%$ in the ALSS group (Table 3).

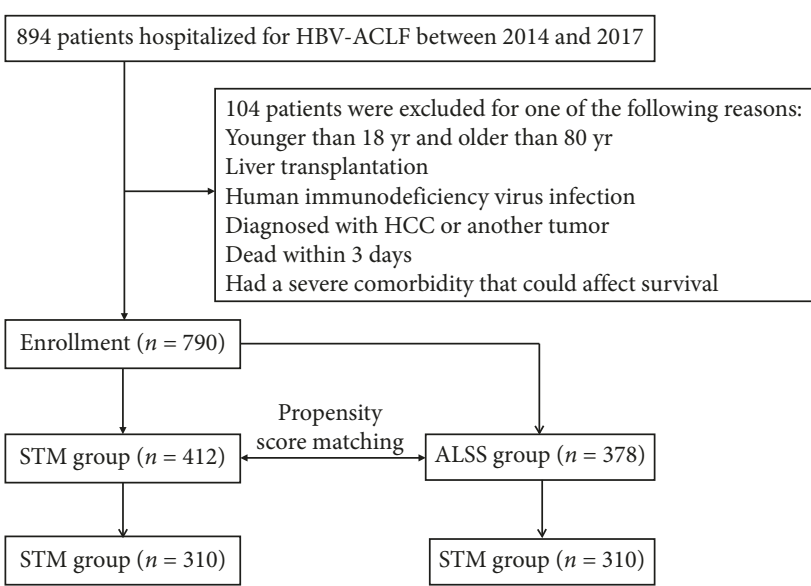

FIGURE 1: Flowchart of the patient selection process. HBV-ACLF, hepatitis B virus-related acute-on-chronic liver failure; LT, liver transplantation; STM, standard medical therapy; ALSS, artificial liver support system.

3.3. Changes in Laboratory Parameters at Day 7 and Day 28. The effect of treatment on laboratory parameters at day 7 and day 28 is shown in Tables 4 and 5, respectively. Compared with the SMT treated alone group, treatment with ALSS was associated with a significant reduction in serum T-Bil levels and MELD scores at both day 7 and day 28; however, the serum creatinine only decreased at day 7 . The remaining analyzed parameters showed no significant difference between the two groups.

3.4. Risk Factors on 28-Day Survival. Using multivariate logistic regression analysis, the independent baseline risk factors for 28-day survival were identified as age, T-Bil, low albumin, ALT, MELD score, and COSSH-ACLF grade (Table 6). Cirrhosis, INR, serum creatinine, and platelet count were not independent predictors of 28-day survival.

\section{Discussion}

HBV-ACLF is observed in populations with HBV-related chronic liver disease. Liver transplantation is the most effective therapy for patients with liver failure; however, less than $30 \%$ of patients have access to transplantation because of donor shortages and the extremely poor prognosis of HBV-ACLF [21]. Although NA treatment could effectively decrease the 3-month mortality for patients with HBVACLF, NAs are only valid in patients with MELD scores less than $30[22,23]$. In addition, mutations resistant to NAs are frequent precipitating events of HBV-ACLF, which is related to high mortality [24]. The development of ACLF includes the accumulation of various metabolites and toxins that vary in size, distribution volume, lipophilicity, and proteinbinding abilities $[25,26]$. Most ALSSs are capable of correcting the hemato-microenvironment, such as detoxification, synthesis, immune regulation, and reducing mortality in patients with ACLF, when compared with SMT [27]. Therefore, ALSS has been recommended as an important method to treat ACLF. 
TABLE 1: Clinical characteristics of the two groups studied at baseline.

\begin{tabular}{|c|c|c|c|c|c|c|}
\hline \multirow{2}{*}{ Characteristics } & \multicolumn{3}{|c|}{ Entire cohort } & \multicolumn{3}{|c|}{ Propensity score-matched cohort } \\
\hline & $\operatorname{SMT}(n=412)$ & ALSS $(n=378)$ & $p$ value & $\operatorname{SMT}(n=310)$ & ALSS $(n=310)$ & $p$ value \\
\hline Age $(y)$ & $44.0(10.6)$ & $46.9(11.4)$ & 0.097 & $45.4(11.1)$ & $46.8(11.3)$ & 0.286 \\
\hline Sex, male & $351(85.2)$ & $337(86.0)$ & 0.097 & $271(87.4)$ & $274(84.6)$ & 0.712 \\
\hline HBV-DNA (log copies/mL) & $5.1(1.6)$ & $5.1(2.0)$ & 0.641 & $5.2(1.1)$ & $5.1(1.9)$ & 0.705 \\
\hline Alanine transaminase level (IU/L) & $388.5(277.4)$ & $341.1(326.5)$ & 0.010 & $376.3(239.8)$ & $360.4(300.1)$ & 0.695 \\
\hline Total bilirubin level (mg/dl) & $22.1(7.4)$ & $24.4(7.0)$ & 0.002 & $22.7(7.4)$ & $23.9(7.0)$ & 0.121 \\
\hline Serum albumin level $(\mathrm{g} / \mathrm{L})$ & $33.1(5.9)$ & $32.1(6.3)$ & 0.027 & $33.1(5.6)$ & $31.9(5.4)$ & 0.149 \\
\hline Platelet count $\left(\times 105 / \mathrm{mm}^{3}\right)(\mathrm{SD})$ & $107.3(56.3)$ & $103.8(48.1)$ & 0.015 & $105.3(57.9)$ & $104.7(45.8)$ & 0.893 \\
\hline White cell count $(\times 109 / \mathrm{L})$ & $9.1(21.3)$ & $7.9(3.3)$ & 0.018 & $9.4(24.1)$ & $7.6(4.0)$ & 0.197 \\
\hline Serum creatinine level (mg/dl) & $82.3(44.3)$ & $76.5(30.6)$ & 0.006 & $72.6(23.7)$ & $68.5(25.5)$ & 0.088 \\
\hline International normalized ratio & $2.5(0.9)$ & $2.5(0.6)$ & 0.759 & $2.3(0.8)$ & $2.4(0.9)$ & 0.687 \\
\hline Serum sodium $(\mathrm{mEq} / \mathrm{L})$ & $137.6(4.5)$ & $136.0(4.1)$ & $<0.001$ & $135.7(4.9)$ & $136.4(3.7)$ & 0.181 \\
\hline Hepatic encephalopathy $\geq$ grade II & $9.5 \%(39 / 412)$ & $13.8 \%(52 / 378)$ & 0.059 & $10.3 \%(32 / 310)$ & $14.2 \%(44 / 378)$ & 0.142 \\
\hline Cirrhosis & $158(38.3)$ & $161(42.6)$ & 0.225 & $118(38.1)$ & $133(42.9)$ & 0.220 \\
\hline MELD score & $25.9(5.2)$ & $24.8(5.6)$ & 0.721 & $25.1(5.2)$ & $24.9(5.6)$ & 0.594 \\
\hline COSSH-ACLF grade & & & 0.091 & & & 0.985 \\
\hline ACLF grade 1 & $226(54.9)$ & $235(62.2)$ & & $207(66.8)$ & $209(67.4)$ & \\
\hline ACLF grade 2 & $174(42.2)$ & $131(34.7)$ & & $94(30.3)$ & $92(29.7)$ & \\
\hline ACLF grade 3 & $12(2.9)$ & $12(3.2)$ & & $9(2.9)$ & $9(2.9)$ & \\
\hline Nucleos $(\mathrm{t})$ ide analogues & & & $<0.001$ & & & $<0.001$ \\
\hline Lamivudine & 205 & 167 & & 155 & 131 & \\
\hline Entecavir & 207 & 182 & & 155 & 157 & \\
\hline Tenofovir & 0 & 18 & & 0 & 14 & \\
\hline Telbivudine & 0 & 11 & & 0 & 8 & \\
\hline
\end{tabular}

Values are expressed as number and percentage or mean \pm SD unless otherwise specified. SMT, standard medical therapy; ALSS, artificial liver support system; MELD, model for end-stage liver disease; COSSH-ACLF, Chinese Group on the Study of Severe Hepatitis B-ACLF.

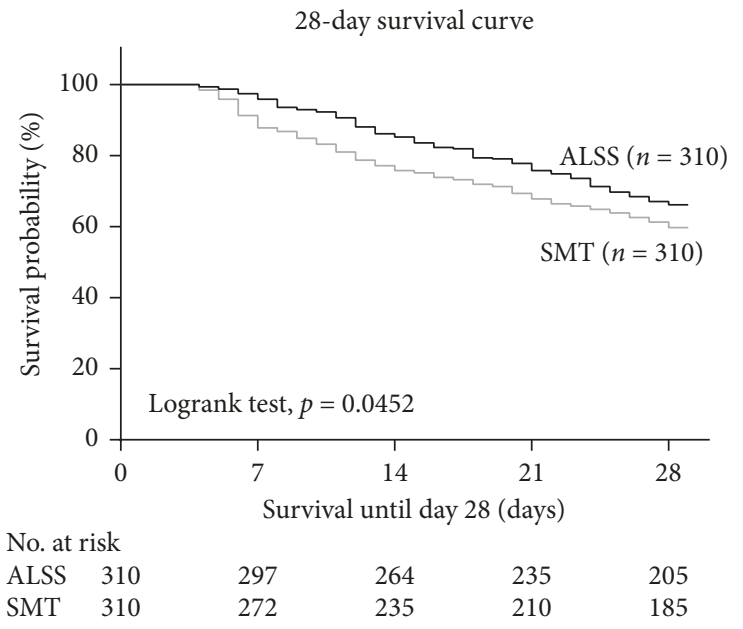

(a)

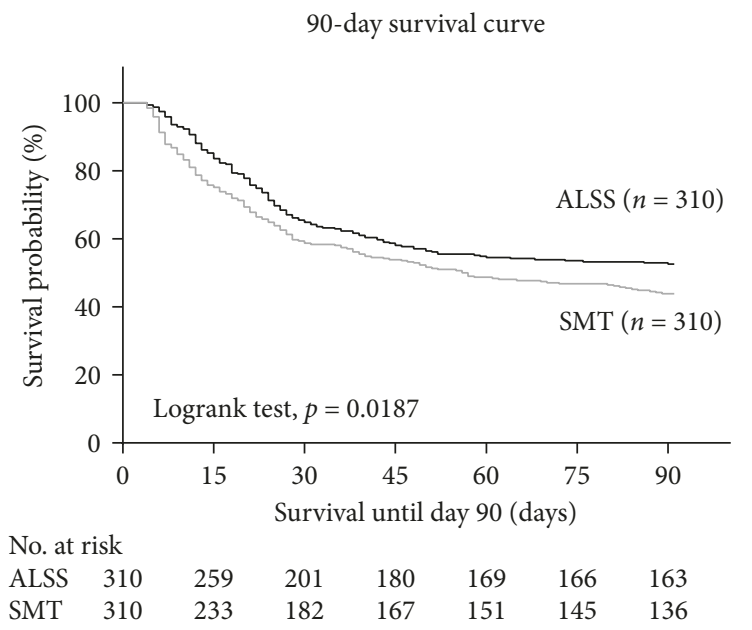

(b)

FIGURE 2: Short-term (28/90 days) survival curves for patients with HBV-ACLF. (a) 28-day survival curves for patients in the SMT and ALSS groups. (b) 90-day survival curves for patients in the SMT and ALSS groups. HBV-ACLF, hepatitis B virus-related acute-on-chronic liver failure; STM, standard medical therapy; ALSS, artificial liver support system.

HBV-ACLF is a special type of ACLF and is the most frequent ACLF in China. This large, multicenter, nationwide, historical retrospective study showed that treatment with ALSS in patients with HBV-ACLF remarkably improved their short-term (28/90 days) survival rates compared with those receiving SMT only. The results indicated that ALSS is effective at removing the toxic substances from plasma that accumulate in patients with HBV-ACLF (confirmed by the significant decreased in T-Bil), correcting coagulopathy (confirmed by the INR decline), and alleviating renal failure (confirmed by the creatinine decline). The functions of synthesis and immune regulation have been well established in pigs with ALF, although further clinical studies are needed.

The ALSS performed in this study innovatively used plasma separators with an aperture of about $1 / 10$ 
TABLE 2: Short-term (28/90 days) survival rates for patients treated with lamivudine vs entecavir or patients with noncirrhotic HBV-ACLF vs cirrhotic HBV-ACLF.

\begin{tabular}{lcccccc}
\hline \multirow{2}{*}{ Nucleos(t)ide analogues } & \multicolumn{3}{c}{ 28-day survival rate } & \multicolumn{3}{c}{90 -day survival rate } \\
& SMT & ALSS & Overall & SMT & ALSS & Overall \\
\hline Lamivudine & $61.3 \%(95 / 155)$ & $64.1 \%(84 / 131)$ & $62.6 \%(179 / 286)$ & $41.9 \%(65 / 155)$ & $49.6 \%(65 / 131)$ & $45.5(130 / 286)$ \\
Entecavir & $58.1 \%(90 / 155)$ & $77.1 \%(121 / 157)$ & $67.6 \%(211 / 312)$ & $45.8 \%(71 / 155)$ & $62.4 \%(98 / 157)$ & $54.2 \%(169 / 312)$ \\
$p$ value & 0.563 & 0.016 & 0.794 & 0.492 & 0.029 & 0.033 \\
Noncirrhotic HBV-ACLF & $60.4 \%(116 / 192)$ & $67.0 \%(120 / 179)$ & $63.6 \%(236 / 371)$ & $45.3 \%(87 / 192)$ & $54.2 \%(97 / 179)$ & $49.6 \%(184 / 371)$ \\
Cirrhotic HBV-ACLF & $58.5 \%(69 / 118)$ & $64.9 \%(85 / 131)$ & $61.8 \%(154 / 249)$ & $41.5 \%(49 / 118)$ & $50.4 \%(66 / 131)$ & $46.2 \%(115 / 249)$ \\
$p$ value & 0.735 & 0.692 & 0.656 & 0.514 & 0.507 & 0.405 \\
\hline
\end{tabular}

TABLE 3: Short-term survival rates for patients in different COSSH-ACLF grades.

\begin{tabular}{cccc}
\hline COSSH-ACLF & Prevalence & 28-day survival rate & 90 -day survival rate \\
\hline Total $(n=790)$ & & $60.0 \%(474 / 790)$ & $45.8 \%(362 / 790)$ \\
ACLF grade 1 & $58.4 \%(461 / 790)$ & $76.1 \%(351 / 461)$ & $61.2 \%(282 / 461)$ \\
ACLF grade 2 & $38.6 \%(305 / 790)$ & $40.0 \%(122 / 305)$ & $26.2 \%(80 / 305)$ \\
ACLF grade 3 & $3.0 \%(24 / 790)$ & $4.2 \%(1 / 24)$ & $0(0 / 24)$ \\
SMT $(n=310)$ & & $59.0 \%(185 / 310)$ & $42.3 \%(136 / 310)$ \\
ACLF grade 1 & $66.8 \%(207 / 310)$ & $72.5 \%(150 / 207)$ & $55.6 \%(115 / 207)$ \\
ACLF grade 2 & $30.3 \%(94 / 310)$ & $37.2 \%(35 / 94)$ & $22.3 \%(21 / 94)$ \\
ACLF grade 3 & $2.9 \%(9 / 310)$ & $0(0 / 9)$ & $0(0 / 9)$ \\
ALSS $(n=310)$ & & $65.2 \%(205 / 310)$ & $51.0 \%(163 / 310)$ \\
ACLF grade 1 & $67.4 \%(209 / 310)$ & $78.5 \%(164 / 209)$ & $65.1 \%(136 / 209)$ \\
ACLF grade 2 & $29.7 \%(92 / 310)$ & $43.5 \%(40 / 92)$ & $29.3 \%(27 / 92)$ \\
ACLF grade 3 & $2.9 \%(9 / 310)$ & $11.1 \%(1 / 9)$ & $0(0 / 9)$ \\
\hline
\end{tabular}

SMT, standard medical therapy; ALSS, artificial liver support system.

TABLE 4: Effects of treatment on laboratory parameters at day 7.

\begin{tabular}{|c|c|c|c|}
\hline Parameter & SMT $(n=272)$ & ALSS $(n=392)$ & $p$ value \\
\hline \multicolumn{4}{|l|}{ Serum bilirubin $(\mathrm{mg} / \mathrm{dl})$} \\
\hline Baseline & $22.8(7.3)$ & $23.6(7.0)$ & \\
\hline Day 7 & $23.9(7.5)$ & $22.2(6.8)$ & \\
\hline Change from baseline & $1.1(7.2)$ & $-1.4(4.7)$ & 0.008 \\
\hline \multicolumn{4}{|l|}{ Serum albumin $(g / L)$} \\
\hline Baseline & $33.6(5.4)$ & $32.1(5.3)$ & \\
\hline Day 7 & $33.8(7.7)$ & $34.1(7.3)$ & \\
\hline Change from baseline & $0.2(4.5)$ & $2.0(17.3)$ & 0.067 \\
\hline \multicolumn{4}{|c|}{ Baseline international normalized ratio } \\
\hline Baseline & $2.3(0.8)$ & $2.4(0.9)$ & \\
\hline Day 7 & $2.2(0.8)$ & $2.2(0.8)$ & \\
\hline Change from baseline & $-0.1(0.50)$ & $-0.2(0.92)$ & 0.064 \\
\hline \multicolumn{4}{|l|}{ Serum creatinine $(\mu \mathrm{mol} / \mathrm{L})$} \\
\hline Baseline & $72.6(23.7)$ & $68.5(25.5)$ & \\
\hline Day 7 & $75.9(28.5)$ & $66.2(24.8)$ & \\
\hline Change from baseline & $3.3(25.2)$ & $-2.3(17.9)$ & 0.043 \\
\hline \multicolumn{4}{|c|}{ Alanine transaminase level (IU/L) } \\
\hline Baseline & $371.8(237.1)$ & $360.4(300.1)$ & \\
\hline Day 7 & $138.4(137.1)$ & $159.7(196.7)$ & \\
\hline Change from baseline & $-233.4(293.7)$ & $-200.7(241.8)$ & 0.071 \\
\hline \multicolumn{4}{|l|}{ MELD score } \\
\hline Baseline & $25.2(5.1)$ & $25.0(5.6)$ & \\
\hline Day 7 & $25.1(4.7)$ & $24.3(5.8)$ & \\
\hline Change from baseline & $-0.1(3.4)$ & $-0.7(6.2)$ & 0.036 \\
\hline
\end{tabular}

SMT, standard medical therapy; ALSS, artificial liver support system; MLED, model for end-stage liver disease.

(membrane pore size $=0.03 \mu \mathrm{m}$ ) of that of a normal plasma separator for direct $\mathrm{PE}$, which could remove toxic substances effectively for patients with liver failure, retain important plasma components, and reduce the plasma dosage [28]. Although high-volume plasma (HVP, approximately $8 \mathrm{~L}$ ) exchange has a beneficial therapeutic effect on patients with 
TABLE 5: Effects of treatment on laboratory parameters at day 28.

\begin{tabular}{|c|c|c|c|}
\hline Parameter & SMT $(n=185)$ & ALSS $(n=205)$ & $p$ value \\
\hline \multicolumn{4}{|l|}{ Serum bilirubin $(\mathrm{mg} / \mathrm{dl})$} \\
\hline Baseline & $22.1(7.1)$ & $23.2(6.8)$ & $<0.001$ \\
\hline Day 28 & $15.2(10.1)$ & $22.8(10.8)$ & \\
\hline Change from baseline & $-4.4(9.5)$ & $-6.9(9.5)$ & \\
\hline \multicolumn{4}{|l|}{ Serum albumin $(g / L)$} \\
\hline Baseline & $33.6(5.4)$ & $32.1(5.3)$ & \\
\hline Day 28 & $33.8(7.7)$ & $34.1(7.3)$ & \\
\hline Change from baseline & $0.2(4.5)$ & $2.0(17.3)$ & 0.067 \\
\hline \multicolumn{4}{|c|}{ Baseline international normalized ratio } \\
\hline Baseline & $2.3(0.6)$ & $2.3(0.6)$ & \\
\hline Day 28 & $1.7(0.7)$ & $1.7(0.7)$ & \\
\hline Change from baseline & $-0.5(0.7)$ & $-0.5(0.7)$ & 0.975 \\
\hline \multicolumn{4}{|l|}{ Serum creatinine $(\mu \mathrm{mol} / \mathrm{L})$} \\
\hline Baseline & $73.2(22.7)$ & $70.9(22.8)$ & \\
\hline Day 28 & $72.4(34.5)$ & $70.8(25.9)$ & \\
\hline Change from baseline & $-0.8(34.8)$ & $-0.1(23.4)$ & 0.088 \\
\hline \multicolumn{4}{|l|}{ ALT (IU/L) } \\
\hline Baseline & $356.2(242.5)$ & $367.3(287.4)$ & \\
\hline Day 7 & $60.8(89.7)$ & $44.9(26.7)$ & \\
\hline Change from baseline & $-295.4(293.7)$ & $-322.4(241.8)$ & 0.085 \\
\hline \multicolumn{4}{|l|}{ MELD score } \\
\hline Baseline & $25.2(4.2)$ & $25.4(4.8)$ & \\
\hline Day 28 & $17.7(9.5)$ & $16.8(7.8)$ & \\
\hline Change from baseline & $-7.5(8.2)$ & $-8.6(6.4)$ & 0.042 \\
\hline
\end{tabular}

SMT, standard medical therapy; ALSS, artificial liver support system; MLED, model for end-stage liver disease.

TABLE 6: Multivariate logistic regression model investigating independent risk factors on 28-day survival.

\begin{tabular}{lccc}
\hline Variable & Reference & OR (CI 95 \%) & $p$ value \\
\hline Age & Per year & $1.017(1.002-1.033)$ & 0.022 \\
Total bilirubin & Per unit increase & $1.062(1.035-1.088)$ & $<0.001$ \\
Alanine transaminase & Per unit increase & $1.001(1.000-1.0001)$ & 0.001 \\
Albumin & Per unit decline & $1.053(1.011-1.074)$ & 0.009 \\
MELD score & Per point increase & $1.059(1.011-1.109)$ & 0.015 \\
COSSH-ACLF grade & Per rank increase & $2.683(1.792-4.017)$ & $<0.001$ \\
\hline
\end{tabular}

MLED, model for end-stage liver disease; COSSH-ACLF, Chinese Group on the Study of Severe Hepatitis B-ACLF.

ALF [26, 29], it is usually performed over more than two sessions for patients with ACLF, which results in a shortage of fresh plasma. Moreover, the HVP removes almost all elements of plasma; part of them are beneficial substances (such as hepatic growth factor) for liver regeneration [30]. In this study, the ALSS using lower-volume plasma (LVP) exchange in which the total volume of exchanged fresh plasma is approximately $1500 \mathrm{~mL}$ [12]. The LVP is usually the first step of the ALSS, and subsequent adsorption and hemofiltration circulation are conducted through autologous plasma derived from waste plasma. The waste plasma is purified, which avoids wasting massive plasma and reduces the loss of essential substances. However, whether lowvolume PE provides better results compared with highvolume PE remains unclear.

Selection of suitable inclusion criteria is crucial to evaluate the efficacy of ALSS for patients with HBV-ACLF. HBV-ACLF is an extremely special type of ACLF with some distinctive characteristics. Patients with HBV-ACLF have poorer prognosis than patients with non-HBV-ACLF (the
$28 / 90$ days mortality rates were $60.2 \%$ vs $52.1 \%$ and $73.9 \%$ vs 69.7\%) [6]. Patients with HBV-ACLF have a higher incidence of liver and coagulation failure [31, 32], whereas kidney failure and cerebral failure are the most common types of organ failure in patients with non-HBV-ACLF. Reactivation of $\mathrm{HBV}$, an acute hepatic insult, is the leading cause of HBV-ACLF in the Asian region [33-36]. The European Association for the Study of the Liver (EASL) criteria and the American Association for the Study of Liver Diseases (AASLD) criteria are the major criteria for ACLF in patients from Europe and North America, where alcoholic liver disease is the major etiology $[7,37]$. These criteria may not apply to China, where hepatitis $B$ virus infection is the major etiology in China. The COSSH criteria were established based on a large Chinese HBV-ACLF group which is in accordance with our study group. Therefore, we chose the COSSH criteria as the inclusion criteria.

A previous study showed that the first leading cause of HBV-ACLF was spontaneous severe acute exacerbation of $\mathrm{CHB}(62.5 \%)$ and the second leading cause was alcohol 
(15.4\%) in Asia [5]. The oral NA therapy effectively suppresses viral DNA and prevents the progression of liver inflammation; therefore, rapid initiation of oral NA treatment in patients with HBV-ACLF is recommended widely $[38,39]$. LAM and ETV are both widely used in China, though LAM has less potency and has higher resistance (LAM: 70\%; ETV: 1.2\%) than ETV [39].

This study revealed that compared with LAM, ETV did not improve the short-term survival rates in HBV-ACLF, neither in the SMT group nor ALSS group. These results were similar to a largest meta-analysis, which demonstrated a comparable short-term mortality (within 4 months) of LAM and ETV; however, ETV revealed a more favorable long-term (beyond 4 months) outcome than LAM in patients with HBV-ACLF [40]. The results of multivariate logistic regression showed that cirrhosis is not a risk-independent predictor of 28-day survival. We also found a similarly short-term survival rate in patients with both cirrhotic HBV-ACLF (61.8\%/46.2\%) and noncirrhotic HBVACLF (63.6\%/49.65\%). However, this result was not in line with that of Wu's study, which indicated that cirrhosis patients had superior 28-day survival rate than noncirrhosis patients (47.9\%: 39.8\%, $p<0.05)$ [6]. The contradictory outcomes might result from small population samples of this study. In previous studies, T-Bil and platelet levels were found as independent risk factors of mortality among HBVACLF patients; however, in our study, the significant difference was only found in single-factor logistic regression analysis.

In conclusion, among patients with HBV-ACLF in China, ALSS has better therapeutic effect than SMT. Though the treatment for HBV-ACLF haas improved over the past three decades the short-term mortality of ACLF remains high. Therefore, more effective therapeutic methods should be investigated.

\section{Data Availability}

All relevant data are within the paper and its supporting information file.

\section{Ethical Approval}

The study was reviewed and approved by the ethics committee of the First Affiliated Hospital, College of Medicine, Zhejiang University.

\section{Conflicts of Interest}

The authors have declared that there are no conflicts of interest.

\section{Authors' Contributions}

Li LJ and Xiao LL designed the research. Xiao LL, Xu XW, Wu XX, Huang KZ, and Zhang LJ performed the research. Xiao LL and Zhao YL analyzed the data. All authors wrote the paper, had access to the study data, and have reviewed and approved the final manuscript.

\section{Acknowledgments}

This work was supported by the Science \& Technology Key Program of Zhejiang China (\#2017C03051).

\section{References}

[1] S. Zhang, F. Wang, and Z. Zhang, "Current advances in the elimination of hepatitis B in China by 2030," Frontiers of Medicine, vol. 11, no. 4, pp. 490-501, 2017.

[2] T. Vos, A. A. Abajobir, and K. H. Abate, "Global, regional, and national incidence, prevalence, and years lived with disability for 328 diseases and injuries for 195 countries, 1990-2016: a systematic analysis for the global burden of disease study 2016," The Lancet, vol. 390, no. 10100, pp. 1211-1259, 2017.

[3] S. Nayagam, M. Thursz, E. Sicuri et al., "Requirements for global elimination of hepatitis B: a modelling study," The Lancet Infectious Diseases, vol. 16, no. 12, pp. 1399-1408, 2016.

[4] F.-S. Wang, J.-G. Fan, Z. Zhang, B. Gao, and H.-Y. Wang, "The global burden of liver disease: the major impact of China," Hepatology, vol. 60, no. 6, pp. 2099-2108, 2014.

[5] G.-J. Xie, H.-Y. Zhang, Q. Chen et al., "Changing etiologies and outcome of liver failure in Southwest China," Virology Journal, vol. 13, no. 1, p. 89, 2016.

[6] T. Wu, J. Li, and L. Shao, "Development of diagnostic criteria and a prognostic score for hepatitis B virus-related acute-onchronic liver failure," Hepatology, vol. 67, no. 12, pp. 21812191, 2017.

[7] R. Moreau, R. Jalan, P. Gines et al., "Acute-on-chronic liver failure is a distinct syndrome that develops in patients with acute decompensation of cirrhosis," Gastroenterology, vol. 144, no. 7, pp. 1426.e9-1437.e9, 2013.

[8] J. Stange, Mitzner, Risler et al., "Molecular adsorbent recycling system (MARS): clinical results of a new membranebased blood purification system for bioartificial liver support," Artificial Organs, vol. 23, no. 4, pp. 319-330, 1999.

[9] D. Kapoor, R. Williams, and R. Jalan, "MARS: a new treatment for hepatorenal failure," Gastroenterology, vol. 119, no. 6, pp. 1799-1800, 2000.

[10] K. Rifai, T. Ernst, U. Kretschmer et al., "Prometheus ${ }^{\circledR}$ — a new extracorporeal system for the treatment of liver failure," Journal of Hepatology, vol. 39, no. 6, pp. 984-990, 2003.

[11] P. Evenepoel, W. Laleman, A. Wilmer et al., "Detoxifying capacity and kinetics of prometheus ${ }^{\circledR}$ - a new extracorporeal system for the treatment of liver failure," Blood Purification, vol. 23, no. 5, pp. 349-358, 2005.

[12] L. J. Li, Artificial Liver, Zhejiang University Press, Hangzhou, China, 2012.

[13] R. Banares, F. Nevens, F. S. Larsen et al., "Extracorporeal albumin dialysis with the molecular adsorbent recirculating system in acute-on-chronic liver failure: the RELIEF trial," Hepatology, vol. 57, no. 3, pp. 1153-1162, 2013.

[14] N. Zhou, J. Li, Y. Zhang et al., "Efficacy of coupled low-volume plasma exchange with plasma filtration adsorption in treating pigs with acute liver failure: a randomised study," Journal of Hepatology, vol. 63, no. 2, pp. 378-387, 2015.

[15] F. Saliba, C. Camus, F. Durand et al., "Predictive factors of transplant free survival in patients with fulminant and subfulminant liver failure: results from a randomized controlled multicenter trial," Journal of Hepatology, vol. 50, pp. S89-S90, 2009.

[16] D. Inderbitzin, B. Muggli, A. Ringger et al., "Molecular absorbent recirculating system for the treatment of acute liver 
failure in surgical patients," Journal of Gastrointestinal Surgery, vol. 9, no. 8, pp. 1155-1162, 2005.

[17] A. Kribben, G. Gerken, S. Haag et al., "Effects of fractionated plasma separation and adsorption on survival in patients with acute-on-chronic liver failure," Gastroenterology, vol. 142, no. 4, pp. 782.e3-789.e3, 2012.

[18] G. Qin, J. G. Shao, B. Wang et al., "Artificial liver support system improves short- and long-term outcomes of patients with HBV-associated acute-on-chronic liver failure: a singlecenter experience," Medicine (Baltimore), vol. 93, no. 28, p. e338, 2014.

[19] A. S. Lok and B. J. McMahon, "Chronic hepatitis B: update 2009," Hepatology, vol. 50, no. 3, pp. 661-662, 2009.

[20] Chinese Medical Association, "Diagnostic and treatment guideline for liver failure," Chinese Journal of Infectious Diseases, vol. 31, no. 3, pp. 129-137, 2013.

[21] W. R. Kim, J. M. Smith, M. A. Skeans et al., "OPTN/SRTR 2012 annual data report: liver," American Journal of Transplantation, vol. 1, pp. 69-96, 2014.

[22] T. Chen, Y. He, X. Liu et al., "Nucleoside analogues improve the short-term and long-term prognosis of patients with hepatitis B virus-related acute-on-chronic liver failure," Clinical and Experimental Medicine, vol. 12, no. 3, pp. 159164, 2012.

[23] L.-J. Sun, J.-W. Yu, Y.-H. Zhao, P. Kang, and S.-C. Li, "Influential factors of prognosis in lamivudine treatment for patients with acute-on-chronic hepatitis B liver failure," Journal of Gastroenterology and Hepatology, vol. 25, no. 3, pp. 583-590, 2010.

[24] F. Zoulim and S. Locarnini, "Hepatitis B virus resistance to nucleos(t)ide analogues," Gastroenterology, vol. 137, no. 5, pp. 1593-1608, 2009.

[25] W. Bernal and J. Wendon, "Acute liver failure," The New England Journal of Medicine, vol. 369, no. 26, pp. 2525-2534, 2013.

[26] W. M. Lee, R. T. Stravitz, and A. M. Larson, "Introduction to the revised American association for the study of liver diseases position paper on acute liver failure 2011," Hepatology, vol. 55, no. 3, pp. 965-967, 2012.

[27] L. L. Kjaergard, J. Liu, B. Nielsen, and C. Gluud, "Artificial and bioartificial support systems for acute and acute-on-chronic liver failure: a systematic review," JAMA, vol. 289, no. 2, pp. 217-222, 2003.

[28] L. J. Li, X. L. Liu, X. W. Xu et al., "Comparison of plasma exchange with different membrane pore sizes in the treatment of severe viral hepatitis," Therapeutic Apheresis and Dialysis, vol. 9, no. 5, pp. 396-401, 2005.

[29] J. Kondrup, T. Almdal, H. Vilstrup, and N. Tygstrup, "High volume plasma exchange in fulminant hepatic failure," The International Journal of Artificial Organs, vol. 15, no. 11, pp. 669-676, 1992.

[30] S. Eguchi, N. Sugiyama, Y. Kawazoe et al., "Total blood exchange suppresses the early stage of liver regeneration following partial hepatectomy in rats," Journal of Artificial Organs, vol. 22, no. 10, pp. 847-853, 1998.

[31] H. Li, L. Y. Chen, N. N. Zhang et al., "Characteristics, diagnosis and prognosis of acute-on-chronic liver failure in cirrhosis associated to hepatitis B," Scientific Reports, vol. 6, no. 1, 2016.

[32] Y. Shi, Y. Yang, Y. Hu et al., "Acute-on-chronic liver failure precipitated by hepatic injury is distinct from that precipitated by extrahepatic insults," Hepatology, vol. 62, no. 1, pp. 232-242, 2015.
[33] S. G. Lim, C. T. Wai, A. Rajnakova, T. Kajiji, and R. Guan, "Fatal hepatitis B reactivation following discontinuation of nucleoside analogues for chronic hepatitis B," Gut, vol. 51, pp. 597-599, 2002.

[34] M. Huang and L. Huang, "Reactivation of hepatitis C viral infection after treatment with infliximab," Journal of Clinical Gastroenterology, vol. 48, no. 2, pp. 189-190, 2014.

[35] E. Sagnelli, M. Pisaturo, M. Stanzione et al., "Clinical presentation, outcome, and response to therapy among patients with acute exacerbation of chronic hepatitis C," Clinical Gastroenterology and Hepatology, vol. 11, no. 9, pp. 1174.e11-1180.e11, 2013.

[36] R. K. Magalhães, D. Ferreira, M. Salgado, and I. Pedroto, "Hepatitis B virus reactivation after chemotherapy in an HIVpositive patient," Journal of Clinical Gastroenterology, vol. 47, no. 6, pp. 565-566, 2013.

[37] S. K. Sarin, C. K. Kedarisetty, Z. Abbas et al., “Acute-onchronic liver failure: consensus recommendations of the asian pacific association for the study of the liver (APASL) 2014," Hepatology International, vol. 8, no. 4, pp. 453-471, 2014.

[38] Y. F. Liaw, J. H. Kao, T. Piratvisuth et al., "Asian-Pacific consensus statement on the management of chronic hepatitis B: a 2012 update," Hepatology International, vol. 6, pp. 531561, 2012.

[39] European Association for the Study of the Liver, "EASL clinical practice guidelines: management of chronic hepatitis B virus infection," Journal of Hepatology, vol. 57, no. 1, pp. 167-185, 2012.

[40] K.-W. Huang, K.-W. Tam, J.-C. Luo, and Y.-C. Kuan, "Efficacy and safety of lamivudine versus entecavir for treating chronic hepatitis B virus-related acute exacerbation and acute-on-chronic liver failure: a systematic review and metaanalysis," Journal of Clinical Gastroenterology, vol. 51, no. 6, pp. 539-547, 2017. 


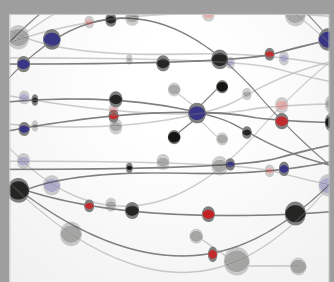

The Scientific World Journal
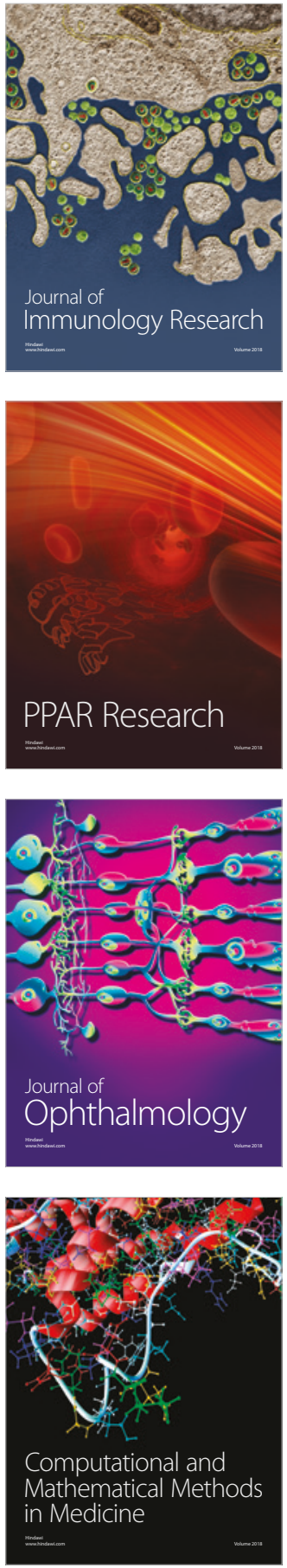

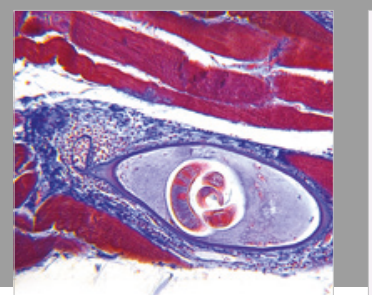

Gastroenterology Research and Practice

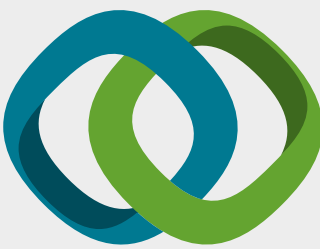

\section{Hindawi}

Submit your manuscripts at

www.hindawi.com
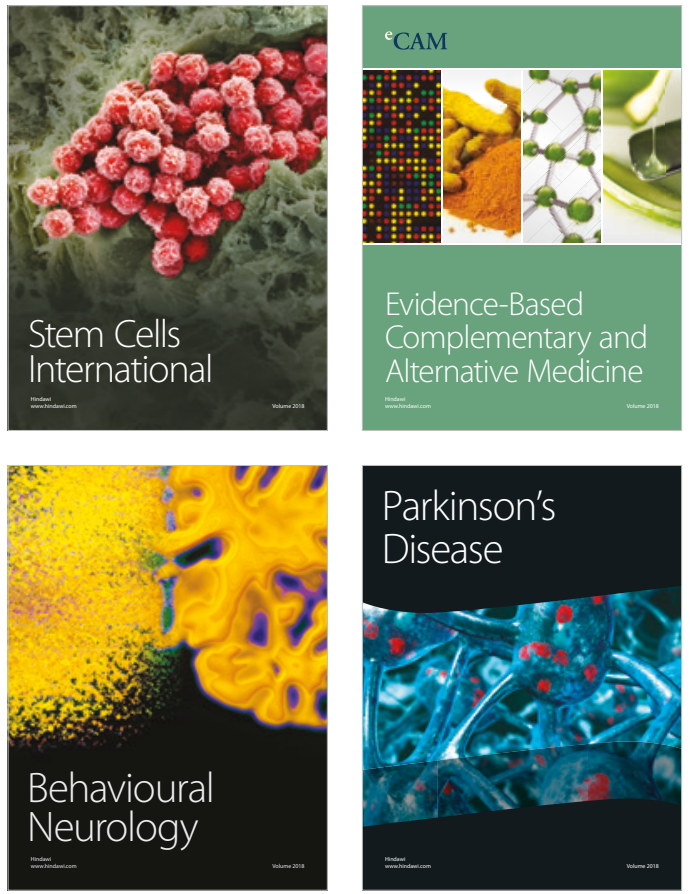

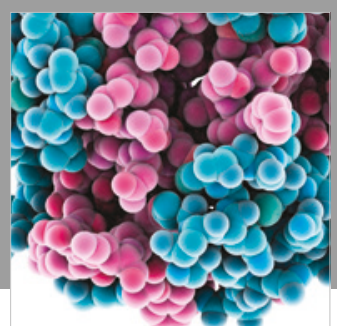

ournal of

Diabetes Research

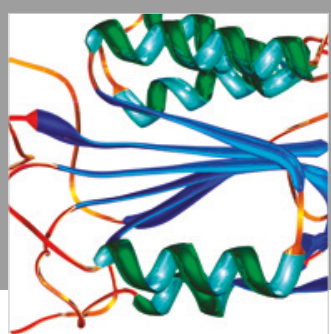

Disease Markers
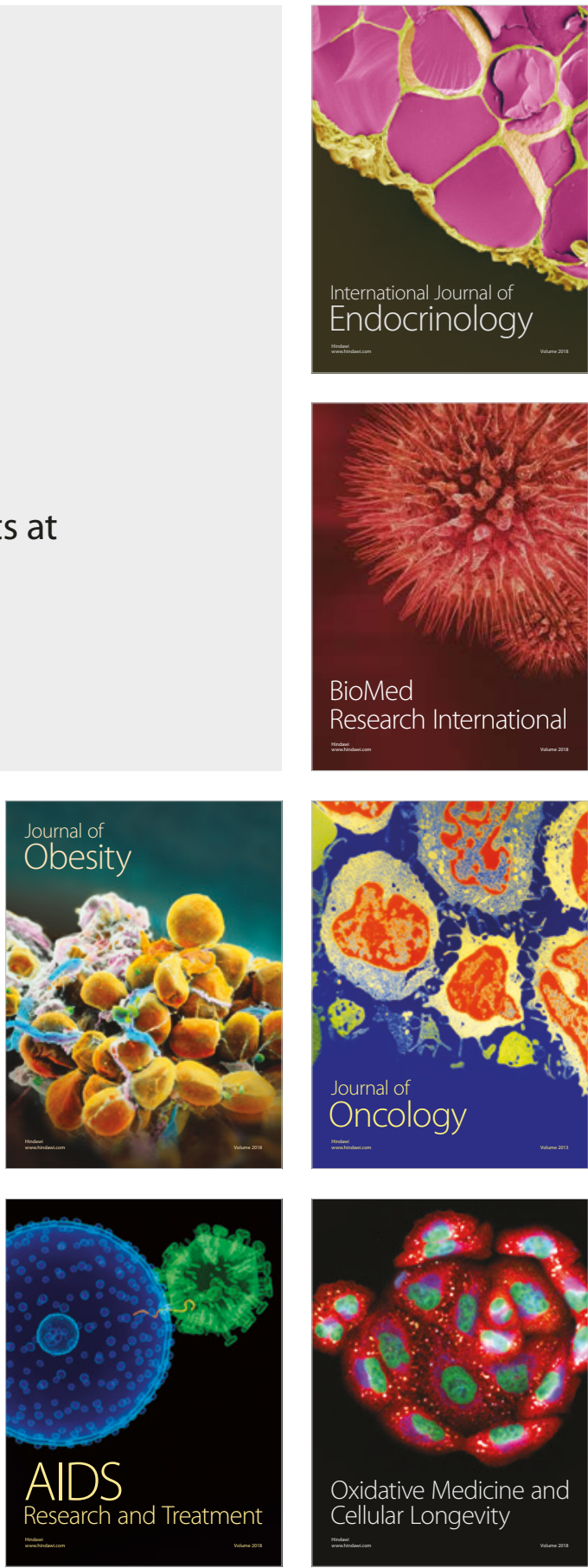